\title{
Investigation and Research on User Satisfaction of Cainiao Courier Station of Sichuan University of Arts and Sciences
}

\author{
Lu Liu1, Zibiao Cheng1, Wanlan Yang1, Li Yang1, Ledan Wang1, Chenxi Huang1, Xiaomei Pu² \\ ${ }^{1}$ School of Finance and Economics Management, Sichuan University of Arts and Science, Dazhou, China \\ ${ }^{2}$ Research Center of Sichuan Old Revolutionary Areas Development, Sichuan University of Arts and Science, Dazhou, China \\ Email: 47872614@qq.com
}

How to cite this paper: Liu, L., Cheng, Z. B., Yang, W. L., Yang, L., Wang, L. D., \& Huang, C. X., \& Pu, X. M. (2020). Investigation and Research on User Satisfaction of Cainiao Courier Station of Sichuan University of Arts and Sciences. Voice of the Publisher, 6, 150-159.

https://doi.org/10.4236/vp.2020.63017

Received: September 3, 2020

Accepted: September 26, 2020

Published: September 29, 2020

Copyright $\odot 2020$ by author(s) and Scientific Research Publishing Inc. This work is licensed under the Creative Commons Attribution International License (CC BY 4.0).

http://creativecommons.org/licenses/by/4.0/

\begin{abstract}
On the basis of understanding the development process of Cainiao Courier station of Sichuan University of Arts and Science, this paper establishes the evaluation index system of user satisfaction, and concludes that the main problems affecting the user satisfaction of Cainiao Courier station of Sichuan University of Arts and Science are the floor space and the arrangement of goods, poor store environment and low rate of warehouse-out equipment. According to the results of this evaluation model, the strategies to improve the user satisfaction of Cainiao Courier Station of Sichuan University of Arts and Sciences are put forward as follows: first, increase the using area of post stations; second, improve the service efficiency of post stations; third, establish two-way feedback platform of post stations; fourth, improve the special situation handling mechanism of post stations; and last, hold "green express" activities.
\end{abstract}

\section{Keywords}

Cainiao Courier Station, Colleges and Universities Express, Evaluation Index System, Degree of Satisfaction

\section{Introduction}

On May 28, 2013, Alibaba led the establishment of Cainiao Network Technology Co., Ltd. and launched the "Cainiao Courier station" project in cooperation with the top 10 logistics companies. At present, more than 1000 Cainiao campus Courier stations have been established across the country, providing an effective solution to the problem of terminal logistics and "the last kilometer" distribution in colleges and universities (Guo \& Li, 2019). With the rapid development of the 
Internet, the requirements of teachers and students for express supermarkets are gradually increasing, and their satisfaction is gradually decreasing. Therefore, it is urgent and important to study the customer satisfaction of Cainiao Courier station in Sichuan University of Arts and Sciences.

\section{Development History and Current Situation to Cainiao Courier Station of Sichuan University of Arts and Sciences}

In 2015, Ling Xiang (the person now in charge of the Cainiao Courier station), contracted to build Cainiao Courier station in the area on the negative first floor of the Music Square of the university, and Cainiao Courier Station of Sichuan University of Arts and Science was formally established. After recent years of development, Cainiao Courier Station of the University cooperated with Boda Assets Management of Sichuan Arts and Sciences Co., LTD., and reached cooperation with common Courier companies in the market, so as to gradually standardize the campus Courier service (Guo, 2016).

Cainiao Courier Station of Sichuan University of Arts and Sciences covers an area of 265 square meters, including 225 square meters of delivery area, 15 square meters of receiving area, 10 square meters of storage room and 15 square meters of office. Its maximum storage capacity is 6000 orders. The shelves in the delivery area include 18 groups of same-day delivery area with a storage area of 259 square meters, and 7 groups of one-day-ago delivery area with a storage area of 53 square meters as well as one group of two-day-ago delivery area with a storage area of 13.5 square meters. The full-time staff of Cainiao Courier Station includes five full-time staff in the new district, one manager and one full-time staff in the old district. Part-time employees include four in the new district and one in the old district. The facilities and equipment of Cainiao Courier Station include 6 handguns, which are used for storehouse and warehouse-out operation; four computers for self-checking, retrieving and monitoring, mailing areas and office areas; one printer, 12 surveillance cameras and one automatic scanning machine for warehouse outing. As shown in Figure 1, Cainiao Courier Station of Sichuan University of Arts and Sciences received an average of 80,000 to 90,000 warehousing entries per month in the first half of the year, and 110,000 to 120,000 warehousing entries per month in the second half.

\section{Construction of Customer Satisfaction Evaluation Index System of Cainiao Courier Station in Sichuan College of Arts and Sciences}

According to the user satisfaction survey of Cainiao Courier Station of Sichuan University of Arts and Science, the factors affecting the satisfaction of Cainiao Courier Station are divided into three levels in combination with the existing research results: first, the target level G is the users' degree of satisfaction of Sichuan University of Arts and Science; the second is the standard layer U, which is the main factor of inbound and outbound management, customer service, 


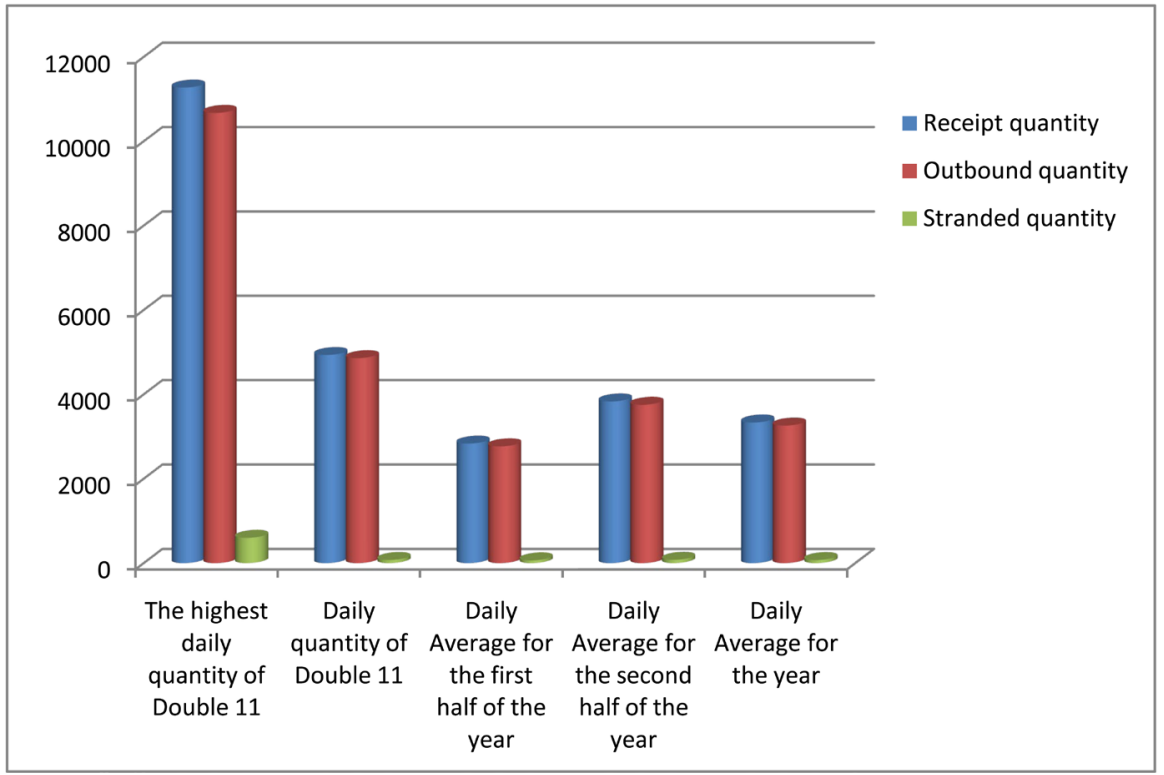

Figure 1. Average daily express volume at Cainiao Post Station of Sichuan University of Arts and Sciences.

geographical location, facilities and equipment; the third is index level $\mathrm{C}$, which is the specific index of the above four factors, the establishment of a hierarchical index system.

\subsection{Users' Satisfaction Evaluation of Cainiao Courier Station of Sichuan University of Arts and Sciences}

In order to quantify the judgment, it is necessary to quantitatively describe the relative superiority of any two schemes to a criterion. In general, for a single criterion, the comparison of two schemes, can always judge the pros and cons. The AHP method adopts 1 - 9 scale to give comparison scale to different comparison, just like in Table $1 .(2,4,6,8$ is the compromise scale between the upper and lower scales, and can be omitted) (Luo, Zhang, \& Zhang, 2020).

Table 1. Scale method table.

\begin{tabular}{|c|c|}
\hline scale bij & Definition and Description \\
\hline 1 & Two elements are equally important to an attribute \\
\hline 3 & Comparing two elements, one is slightly more important than the other \\
\hline 5 & Comparing two elements, one is obviously more important than the other \\
\hline 7 & Comparing two elements, one is much more important than the other \\
\hline \multirow[t]{2}{*}{9} & Comparing two elements, one is extremely more important than the other \\
\hline & Inverse comparison of two elements \\
\hline
\end{tabular}

Index of matrix consistency judgment C.I. (Consistency Index)

$$
\text { C.I. }=\frac{\lambda_{\max }-n}{n-1}
$$


The ratio of C.I. to average random consistency index R.I. is called the random consistency ratio C.R. (Consistency Ratio).

$$
\text { C.R. }=\frac{\text { C.I. }}{\text { R.I. }}
$$

Through questionnaire survey and field interview, teachers and students of Sichuan University of Arts and Science attach importance to various indicators of Cainiao Courier Station's user satisfaction. Through repeated investigation and research, the judgment matrix of the hierarchy of influencing factors of Cainiao Courier Station's user satisfaction of Sichuan University of Arts and Science is determined as shown in Tables 3-7. In addition, the correlation matrix was tested for consistency according to the analytic hierarchy process (AHP), and the results showed that all the five judgment matrices met the requirements of C.I. $<$ 0.1 and C.R. $<0.1$, indicating that all the five judgment matrices passed the consistency test. After relevant calculation, the weights of relevant indicators are obtained, as shown in Table 2. It can be seen that teachers and students of Sichuan University of Arts and Sciences pay more attention to the speed factor of the way of picking up packages, which also conforms to the demand orientation of teachers and students of universities for the Cainiao Courier Station.

Table 2. Evaluation index system and index weight table.

\begin{tabular}{|c|c|c|}
\hline Object level & Standard level (weight) & Index level (weight) \\
\hline \multirow{11}{*}{$\begin{array}{c}\text { Users' degree of } \\
\text { satisfaction of Sichuan } \\
\text { University of Arts and } \\
\text { Science G }\end{array}$} & \multirow{3}{*}{$\begin{array}{l}\text { In-and-out-of-storage } \\
\text { management U1 (0.454) }\end{array}$} & Speed of pickup C1 (0.635) \\
\hline & & Floor space C2 (0.365) \\
\hline & & \\
\hline & \multirow{3}{*}{$\begin{array}{l}\text { Customer service factor } \\
\text { U2 (0.071) }\end{array}$} & Attitude of staff C3 (0.510) \\
\hline & & Regulation of business hours C4 (0.286) \\
\hline & & Handling of customers' complaints C5 (0.204) \\
\hline & \multirow{3}{*}{$\begin{array}{l}\text { Geographical location } \\
\text { factor U3 }(0.197)\end{array}$} & Store environment C6 (0.151) \\
\hline & & Floor space C7 (0.539) \\
\hline & & Arrangement of goods C8 (0.310) \\
\hline & \multirow{2}{*}{$\begin{array}{c}\text { Facilities and equipment } \\
\text { factor U4 }(0.278)\end{array}$} & Scope of equipment check C9 (0.392) \\
\hline & & Rate of outgoing equipment C10 (0.608) \\
\hline
\end{tabular}

Table 3. The judgment matrix table of user satisfaction G.

\begin{tabular}{ccccc}
\hline G & $\mathrm{U} 1$ & $\mathrm{U} 2$ & $\mathrm{U} 3$ & $\mathrm{U} 4$ \\
\hline $\mathrm{U} 1$ & 1 & 6 & 3 & 3 \\
$\mathrm{U} 2$ & $1 / 6$ & 1 & $1 / 5$ & $1 / 7$ \\
$\mathrm{U} 3$ & $1 / 3$ & 5 & 1 & $1 / 2$ \\
$\mathrm{U} 4$ & $1 / 3$ & 7 & 2 & 1 \\
\hline
\end{tabular}

\subsection{Users' Satisfaction Evaluation of Storehouse and Warehouse-Out Management of Cainiao Courier Station}


Table 4. The judgment matrix table of inbound and outbound management factor U1.

\begin{tabular}{ccc}
\hline U1 & G1 & G2 \\
\hline G1 & 1 & 4 \\
G2 & $1 / 4$ & 1 \\
\hline
\end{tabular}

\subsection{Users' Satisfaction Evaluation of Cainiao Courier Station}

Table 5. The judgment matrix of customer service factor U2.

\begin{tabular}{cccc}
\hline U2 & G3 & G4 & G5 \\
\hline G3 & 1 & 3 & 4 \\
G4 & $1 / 3$ & 1 & 2 \\
G5 & $1 / 4$ & $1 / 2$ & 1
\end{tabular}

\subsection{Users' Satisfaction Evaluation of the Geographical Factor of Cainiao Courier Station}

Table 6. The judgment matrix of geographical location factor U3.

\begin{tabular}{cccc}
\hline U3 & G6 & G7 & G8 \\
\hline G6 & 1 & $1 / 7$ & $1 / 4$ \\
G7 & 7 & 1 & 3 \\
G8 & 4 & $1 / 3$ & 1 \\
\hline
\end{tabular}

\subsection{Users' Satisfaction Evaluation of Facility and Equipment of} Cainiao Courier Station

Table 7. The judgment matrix of facility factor U4.

\begin{tabular}{ccc}
\hline U4 & G9 & G10 \\
\hline G9 & 1 & $1 / 3$ \\
G10 & 3 & 1 \\
\hline
\end{tabular}

\section{Customer Satisfaction Evaluation of Cainiao Courier Station of Sichuan University of Arts and Sciences}

\subsection{The Situation of Questionnaire Survey}

According to the principle of random sampling, this questionnaire was sent to all teachers and students of Sichuan University of Arts and Science in December 2019. A total of 405 questionnaires were issued and 400 valid questionnaires were recovered, with an effective rate of $89 \%$. Male students accounted for $35.5 \%$ (142) and female students for $64.5 \%$ (258), which is consistent with the fact that female students prefer shopping. Among the surveyed personnel, 28\% (112 people) often go to Cainiao Courier Station (more than 8 times per month); Sometimes, those who go to Cainiao Courier Station (5 - 8 times per month) account for $39 \%$ ( 156 people), while those who seldom go to people (0 - 4 times 
per month) account for 33\% (132 people), indicating that teachers and students of Sichuan University of Arts and Science have certain consumption ability. The survey data basically conforms to the shopping characteristics of teachers and students in colleges and universities today.

\subsection{Customer Satisfaction Calculation}

According to the questionnaire data, the user satisfaction of Cainiao Courier Station of Sichuan University of Arts and Science was evaluated by fuzzy comprehensive evaluation method. The evaluation level is set as 5 , that is, $\mathrm{V}=\{\mathrm{V} 1$, $\mathrm{V} 2, \mathrm{~V} 3, \mathrm{~V} 4, \mathrm{~V} 5\}$, and the score of $1-2$ is V1, indicating very dissatisfied; a score of 3 - 4 is V2, indicating dissatisfaction; a score of $5-6$ is V3, indicating general satisfaction; a score of 7 - 8 is V4, indicating satisfaction; a score of 9 - 10 is V5, which means very satisfied. The evaluation grade of each index is shown in Table 7. The secondary matrix is constructed according to the above data, which is represented by $\mathrm{R} 1, \mathrm{R} 2, \mathrm{R} 3$ and $\mathrm{R} 4$ respectively.

The weight set of each index determined by AHP in Table 2 is as follows:

The weight of the first indicator

$\mathrm{W}=[0.454,0.071,0.197,0.278]$

Each index weight value of in-and-out-of-storage management factor

$\mathrm{W} 1=[0.635,0.365]$

Each index weight value of customer service factor

$\mathrm{W} 2=[0.510,0.286,0.204]$

The weight value of all indexes of the geographical location factor is

$\mathrm{W} 3=[0.151,0.539,0.310]$

The weight value of all indicators of facility and equipment factors is

$\mathrm{W} 4=[0.392,0.608]$

According to $\mathrm{B}=\mathrm{W} \cdot \mathrm{R}$, the evaluation vectors of the secondary indexes can be calculated as:

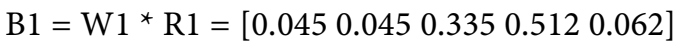

$$
\begin{aligned}
& \mathrm{B} 2=\mathrm{W} 2{ }^{*} \mathrm{R} 2=\left[\begin{array}{lllll}
0.065 & 0.016 & 0.354 & 0.479 & 0.087
\end{array}\right] \\
& \mathrm{B} 3=\mathrm{W} 3 * \mathrm{R} 3=\left[\begin{array}{lllll}
0.048 & 0.050 & 0.427 & 0.342 & 0.132
\end{array}\right] \\
& \mathrm{B} 4=\mathrm{W} 4 \text { * } \mathrm{R} 4=\left[\begin{array}{lllll}
0.052 & 0.073 & 0.423 & 0.371 & 0.081
\end{array}\right]
\end{aligned}
$$

\subsection{Customer Satisfaction Evaluation}

According to the above calculation results, a first-order matrix was established,

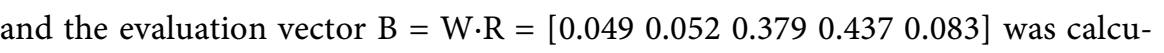
lated and showed that in the overall evaluation of user satisfaction of Cainiao Courier Station of Sichuan University of Arts and Science, $4.9 \%$ of students feel very dissatisfied, $5.2 \%$ feel less satisfied, $37.9 \%$ feel generally satisfied, $43.7 \%$ feel satisfied, and $8.3 \%$ feel very satisfied. In general, Sichuan University of Arts and Science has a high user satisfaction to Cainiao Courier Station, but the proportion of general satisfaction is not small, indicating that there is still a large room for improvement. 
Table 8. The evaluation grade proportion of each index.

\begin{tabular}{|c|c|c|c|c|c|c|}
\hline & Index factors & Very dissatisfied & Dissatisfied & Generally satisfied & Satisfied & Very satisfied \\
\hline \multirow{2}{*}{$\begin{array}{l}\text { In-and-out-of-storage } \\
\text { management }\end{array}$} & Speed of pickup & 0.045 & 0.045 & 0.318 & 0.545 & 0.045 \\
\hline & Floor space & 0.045 & 0.045 & 0.364 & 0.455 & 0.091 \\
\hline \multirow[t]{3}{*}{ Customer service } & Attitude of staff & 0.091 & 0.000 & 0.409 & 0.455 & 0.045 \\
\hline & Regulation of business hours & 0.000 & 0.045 & 0.273 & 0.500 & 0.182 \\
\hline & Handling of customers' complaints & 0.091 & 0.015 & 0.328 & 0.510 & 0.056 \\
\hline \multirow[t]{3}{*}{ Geographic position } & Store environment & 0.045 & 0.051 & 0.404 & 0.369 & 0.131 \\
\hline & Floor space & 0.051 & 0.061 & 0.389 & 0.364 & 0.136 \\
\hline & Arrangement of goods & 0.045 & 0.030 & 0.505 & 0.293 & 0.126 \\
\hline \multirow[t]{2}{*}{ Facility and equipment } & Scope of equipment check & 0.045 & 0.146 & 0.429 & 0.242 & 0.136 \\
\hline & Rate of outgoing equipment & 0.056 & 0.025 & 0.419 & 0.455 & 0.045 \\
\hline
\end{tabular}

From the satisfaction evaluation of teachers and students of Sichuan University of Arts and Sciences on index layer factors, as shown in Table 8, the main problems are the floor space and the arrangement of goods in Cainiao Courier Station, and according to the teachers and students of the surveyed school, the Cainiao Courier Station covers a relatively small area and the arrangement of goods is chaotic, which makes it difficult and crowded for teachers and students to find the goods and thus inefficient (Wang \& Chen, 2018). At the same time, the environment of Courier station stores is not good. In order to save the express cost, the express company uses non-biodegradable plastic bags, which gives off a pungent smell. Although there is a packaging and recycling area in Cainiao Courier Station, some teachers and students do not put the express package into the recycling box of Cainiao Post after taking the package, and throw it into the surrounding or garbage can. If they do not deal with the package in time and encounter strong wind, the phenomenon of packaging bags and waste paper shells flying randomly will appear, which will affect the overall appearance of the school. In addition, due to the low speed of the outgoing equipment, there are often long queues at the peak of the outgoing time and the waiting time is too long. At the same time, it also affects the rate of finding packages for teachers and students, resulting in the greatly reduced efficiency of picking up pieces.

\section{Strategies to Improve Customer Satisfaction of Cainiao Courier Station of Sichuan University of Arts and Sciences}

\subsection{Increase the Using Area of Courier Station}

Although Cainiao Courier Station of Sichuan University of Arts and Sciences covers an area of 265 square meters, due to the large number of teachers and students in the university, the daily storage quantity can reach high to 11,274 pieces and the outbound quantity 106,749 pieces, which requires that Cainiao Courier Station should have enough area to store enough shelves and accom- 
modate large quantities of express delivery. In addition, the number and area of channels also need to be expanded to improve the efficiency of teachers and students in finding items independently, and to avoid stampeding or falling delivery injuries caused by crowding. By increasing the area of use, the capacity of Cainiao Courier Station can also be improved, so as to provide better, more comprehensive and more efficient services for more users who need Cainiao Courier Station of Sichuan University of Arts and Sciences.

\subsection{Improve the Service Efficiency of Courier Station}

The main service objects of Cainiao Post station of Sichuan University of Arts and Sciences are teachers and students. Affected by the unified schedule, the time for picking up samples is relatively unified, which is likely to cause congestion during the picking up process. Service efficiency has become one of the important factors affecting the satisfaction of teachers and students (Yin, Bao, Chen, Yu, Li, \& Wang, 2020). The concrete improvement measures to improve the service efficiency of Cainiao Courier Station are as follows. Improve the efficiency of express delivery warehousing, reasonably plan the warehousing route, and scientifically arrange the warehousing time of each express company, and transport the express delivery to Cainiao Courier Station within the specified time for warehousing operation, so as to avoid the situation that the express delivery on the same day arrives at Cainiao Courier Station the next day (Chen, Liao, Yin, \& Zhong, 2019). At the same time improve the efficiency of express delivery in and out. First, it is necessary to rationally plan the layout of shelf arrangement in Cainiao Courier Station. The shelves should be arranged in sequence of Numbers. Meanwhile, express delivery should be placed according to the classification number of package size and weight, so as to reduce the time for students to find express delivery and improve the efficiency of express delivery in and out (Wang \& Feng, 2019). The second is to simplify the process of express delivery and warehousing and the receiving steps. By taking advantage of the universality of the network, teachers and students can receive the delivery push message by downloading The Cainiao Wrapping APP, and complete the authentication in advance. Teachers and students can place an order through the Cainiao Wrapping APP in advance, and directly give the express mail to the staff to pack when they arrive at the Cainiao Post station (Zou \& Yin, 2019).

\subsection{Establish Two-Way Feedback Platform of Post Stations}

Create a public account of Cainiao Courier Station of Sichuan University of Arts and Science on WeChat platform. Through WeChat public account platform, teachers and students can evaluate the service of Cainiao Courier Station, inquire express information, give feedback and suggestions, and make complaints, so as to vent their dissatisfaction. Teachers, students and the staff of Cainiao Courier Station gradually formed two-way interaction, and the emotions of teachers and students were vented and calmed down, and their satisfaction 
would be gradually improved (Du, 2017).

\subsection{Improve the Special Situation Handling Mechanism of Post Stations}

According to the case of missing express item, the most important problem to be solved is the specification of the operation process of express delivery in and out of the warehouse, so as to avoid the occurrence of less and missed deliveries. At the same time, it is also necessary to strictly verify the information of the pickers, strengthen the accuracy of camera surveillance at Cainiao Courier Station, and avoid the occurrence of wrong packages or stolen packages. If a person is found stealing packages, report the theft to the college or university and discuss with the school to deal with the case. In terms of the intact degree of express delivery, if the express delivery is found damaged, if the damage degree is relatively minor, the packaging should be reinforced in time, and if the damage is serious, the consignee should be immediately notified to come to check and confirm the express delivery, and negotiate with the user on the treatment measures.

\subsection{Hold "Green Express" Activities}

First, Cainiao Courier Station can raise money from various Courier companies to promote and use biodegradable bags. Second, a green campus express day can be set from the school level, with the theme activity of a "create green logistics, start from you and me", to publicize and promote the use of biodegradable packaging through public platforms such as campus WeChat official account, QQ space and WeChat group, so as to raise the awareness of green environmental protection among teachers and students of the school as well as the express delivery industry. Thirdly, the number of recycling bins can be increased to avoid overflowing. We can also sort and recycle other packaging, and cooperate with the paper shell factory near the school to send the paper shell that cannot be directly used to the paper factory for secondary recycling and processing, which can not only make a second use of express package, but also reduce the cost of packaging materials, reduce the pollution of campus environment, save natural resources, and deeply practice the concept of "green logistics" (Wang, Zhao, \& Zhang, 2020).

\section{Conclusion}

Based on the investigation and research on the user satisfaction of the Cainiao Courier Station of Sichuan University of Arts and Sciences, the main factors influencing the user satisfaction of the Cainiao Station of Sichuan University of Arts and Sciences are obtained in this paper, and the concrete and feasible solutions are put forward according to the influencing factors. But as a result of the limitation of time and space, the research of this paper and its results have limitations, which can not apply to every novice station, and as with the rapid development of electronic commerce and express delivery industry, more and 
more people begin to pay close attention to the Cainiao Courier Station, user demand for Cainiao Courier Station will also gradually increase, and the corresponding countermeasures are also needed to refine and improve accordingly.

\section{Funded Project}

Research achievement of 2019 Undergraduate Scientific Research Project of Sichuan University of Arts and Sciences (Project Number: X2019R106).

\section{Conflicts of Interest}

The authors declare no conflicts of interest regarding the publication of this paper.

\section{References}

Chen, C. L., Liao, J. W., Yin, J., \& Zhong, Y. G. (2019). Study on Service Quality problems and Countermeasures of Cainiao Courier station in Dongguan. Modern Business Trade Industry, 40, 32-33.

Du, H. M. (2017). Analysis of Cainiao Post Status Quo and Development Countermeasures. National Circulation Economy, No. 28, 14-15.

Guo, F. X., \& Li, M. Y. (2019). Research on Service Satisfaction of Cainiao Courier station-A Case Study of Kunming City. Logistics Sci-Tech, 42, 23-26.

Guo, X. (2016). Research on the Development Strategy of Cainiao Post Station in S Universities under the E-Commerce Environment. Yunnan: Normal University.

Luo, Y. S., Zhang, Q., \& Zhang, S. (2020). A Study on the Evaluation of Service Quality of Cainiao Courier Station in Colleges and Universities-A Case Study of Guangdong Institute of Technology. Value Engineering, 39, 126-129.

Wang, B. H., Zhao, R., \& Zhang, Z. R. (2020). Discussion on the Feasibility of Green Express in campus Promotion. Legal System and Society, No. 1, 127-128.

Wang, S. Q., \& Chen, L. (2018). How to Better Play the Role of Cainiao Post Station in Logistics Terminal Distribution. Operation and Management, No. 9, 87-89.

Wang, Z. H., \& Feng, Y. Y. (2019). Research on the Development Current Status and Countermeasures of Campus Cainiao Courier Station-Taking Liaoning University of International Business as an Example. Journal of Chifeng University (Chinese Philosophy and Social Science Edition), 40, 130-133.

Yin, S. W., Bao, X. Y., Chen, H. N., Yu, D. C., Li, J., \& Wang, H. W. (2020). Research on the Development Countermeasures of E-Commerce Logistics "The Last Mile"-A Case study of Cainiao Post Station. Modern Marketing (Business Edition), No. 1, 100-101.

Zou, L., \& Yin, H. (2019). The Satisfaction Analysis of College Cainiao Courier Station Based on Social Network. Journal of Shenzhen University (Science and Engineering), $36,317-323$. 